\title{
Single-incision laparoscopic surgery (SILS), radical hysterectomy for early stage cervical cancer, a case report and review of literature
}

\begin{abstract}
Background: Single-Incision Laparoscopic Surgery (SILS) is one of latest innovations in minimally invasive surgery and has several potential applications in gynecologic oncology surgery. We present a case of early stage cervical cancer that was managed by SILS radial hysterectomy and review the techniques of this procedure

Case: The patient was a 48 year old, Gravida 1, Para 1001, who presented for evaluation of postcoital vaginal bleeding for the past four months. The patient reports regular menstruation, menstrual cycles: 4-5/27-30 days with dysmenorrhea. Speculum examination revealed the posterior vaginal fornix was slightly shallow. The cervix was friable mobile, non-tender, hypertrophic and hard, with a $1.5 \mathrm{X} \mathrm{cm}$ cauliflower-like lesion on the lower cervical lip closing the external cervical os.
\end{abstract}

A Pap smear was significant for high grade squamous intraepithelial lesion (HSIL) with squamous cell carcinoma not being excluded. HPV-DNA detection was positive for $12 \mathrm{high}$ risk strains of HPV (although HPV 16 and HPV 18 were both negative). Colposcopy and cervical biopsy identified low differentiation squamous cell carcinoma of the cervix with atypical cell nests distributed in the mucosal tissue.

The patient underwent single site laparoscopic radical hysterectomy, bilateral salpingooophorectomy (BSO) and pelvic lymphadenectomy. Pathology specimen included a normal uterus and bilateral adnexa with a cauliflower-like cervical mass. Histologic evaluation confirmed the diagnosis of cervical squamous cell carcinoma (non-keratinized), Stage IB1. The patient remained in the hospital for 10 days (standard for this institution) without complication before being discharged home.

Conclusion: Previously, cervical cancer has been managed through LEEP, cone biopsy and total abdominal hysterectomy. Typically, total abdominal hysterectomy surgery has been managed through the laparoscopic approach. Based on our research, a less invasive and perhaps more desired procedure, SILS has been proposed as a safer alternative. SILS uses a single incision site and is associated with less scarring and shortened postoperative hospitalization. More studies are necessary to determine the complete long-term effectiveness of SILS for cervical cancer management. ${ }^{1}$ The good news is gynecologists will find mastering the SILS procedures is well within their current surgical skill set.

Keywords: cervical cancer, laparoendoscopic single-site surgery (LESS), radical hysterectomy, single-incision laparoscopic surgery (SILS), single port laparoscopic radical hysterectomy
Volume 9 Issue 5 - 2018

\author{
Shadi Rezai, ${ }^{1,5}$ Chunyan Zeng, ${ }^{3}$ Alexander C \\ Hughes, ${ }^{2}$ Heather Bergdahl, ${ }^{2}$ Cassandra E \\ Henderson, ${ }^{4}$ Xiaoming Guan, ${ }^{5}$ Juan Liu ${ }^{3}$ \\ 'Department of Obstetrics and Gynecology, Southern California \\ Kaiser Permanente, USA \\ ${ }^{2}$ St. George's University, School of Medicine, St. George's, \\ Grenada \\ ${ }^{3}$ Key Laboratory for Major Obstetric Diseases of Guangdong \\ Province, Department of Obstetrics and Gynecology, The Third \\ Affiliated Hospital of Guangzhou Medical University, China \\ ${ }^{4}$ Maternal and Fetal Medicine, Department of Obstetrics and \\ Gynecology, Lincoln Medical and Mental Health Center, USA \\ ${ }^{5}$ Division of Minimally Invasive Gynecologic Surgery, \\ Department of Obstetrics and Gynecology, Baylor College of \\ Medicine, USA
}

\section{Correspondence: Juan Liu MD PhD, Department of Obstetrics and Gynecology, The Third Affiliated Hospital of Guangzhou Medical University, Guangzhou 5 0 150, China, Tel $+86-20-81292697, \mathrm{Fax}+86-20-81292697$ Email liwjuan900II@163.com}

Xiaoming Guan MD PhD, Section Chief and Fellowship Director, Division of Minimally Invasive Gynecologic Surgery, Department of Obstetrics and Gynecology, Baylor College of Medicine, 665I Main Street, I0th Floor, Houston, Texas, 77030, USA, Tel (832) 826-7464, Fax (832) 825-9349, Email xiaomeng@bcm.edu

Received: July 09, 2018 | Published: October 22, 2018

\section{Background}

Cervical carcinoma (CIN) is typically detected through pathological tissue changes at the squamous-columnar epithelial transition zone. As the disease advances, it may extend into the uterine cavity, vaginal canal and pelvis. Cancer screen is routinely done via Papanicolaou smear test. In a majority of cases where pre-cancerous lesions (CIN Stages I, II, and III) are detected by cytology, associated signs and symptoms are absent. Usually, once symptoms present, the disease has spread extensively. ${ }^{2-4}$

Risk factors for cervical cancer include tobacco use, immunocompromised status, family history of cervical cancer, sexual contact without barrier protection, low social economic status and multiple full-term pregnancies. Additionally, a known causative agent for cervical cancer is human papillomavirus (HPV). In order to avoid contributoryeffects fromsuchriskfactorsand causativeagents, clinicians recommend regular cervical cytologic screening, completing the HPV immunization series, routine condom use, and smoking cessation. ${ }^{3-6}$ Treatment and diagnosing modalities used in current practice for cervical carcinoma include cone biopsy, loop electrosurgical excision procedure (LEEP), laser and cold knife conization and simple hysterectomy. If the cervical malignancy $r$ is found to be in an advanced stage, it may be necessary to perform a radical hysterectomy via laparotomy or laparoscopy with concomitant localized radiation therapy and/or chemotherapy for widespread disease. ${ }^{1,5,7}$ Traditionally, cervical cancer was managed by the previously mentioned techniques; with advancements the Single-Incision Laparoscopic Surgery (SILS) has a high efficacy rate with a minimally invasive approach. ${ }^{1,8,9}$

Single-Incision Laparoscopic Surgery (SILS) is one of latest innovations in minimally invasive surgery and has several potential 
applications in oncologic-gynecologic surgery. SILS should be considered for the treatment of any women with cervical cancer when the extent of invasiveness is minimal. Minimally invasive surgery is becoming a more popular option for many gynecological surgeries due to the decreased blood loss and shortened post-operative stay. ${ }^{1,8}$ As such, we present a case of early stage cervical cancer that was managed by SILS radial hysterectomy. ${ }^{10}$

\section{Presentation of the case}

The patient was a 48-year-old, Gravida 1, Para 1001, who presented for evaluation of new onset of post-coital vaginal bleeding during the previous four months. The patient reports regular menstruation, menstrual cycles: occur regularly between 27-30 days and lasting 4-5 days with dysmenorrhea.

On speculum examination the posterior vaginal fornix was slightly shallow. The vaginal walls were smooth and there were no obvious nodules or lesions. The cervix was mobile, non-tender, hypertrophic and hard, with a $1.5 \times 1 \mathrm{~cm}$ cauliflower-like lesion on the lower cervical lip closing the external cervical os. The lesion was friable, bleeding easily on palpation.

A Pap smear report indicated a high grade squamous intraepithelial lesion (HSIL) with squamous cell carcinoma not being excluded. HPV-DNA detection was positive for 12 high risk strains of HPV (although HPV 16 and HPV 18 were both negative).

Colposcopy and cervical biopsy specimens identified a low differentiation squamous cell carcinoma of the cervix with atypical cell nests distributed in the mucosal tissue.
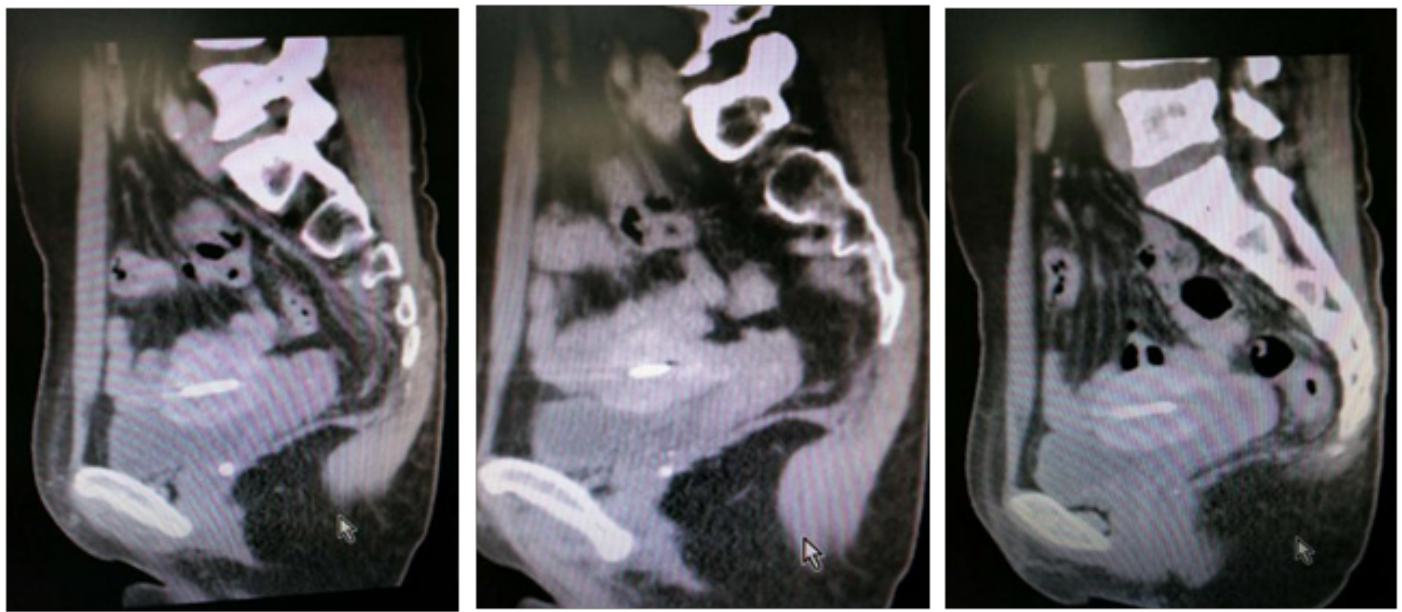

Figure 2 Pelvic CT: showing an enlarged thickened heterogenous cervix measuring $4.1 \times 3.6 \times 3.0 \mathrm{~cm}$, with unclear boundaries. Uterus, bilateral adnexa, bladder, rectum appeared normal with no evidence of pelvic lymphadenopathy, pleural effusion. There are many round high density shadows in the pelvic cavity, diameter of the largest of round high density shadows was about $1.0 \mathrm{~cm}$. Intrauterine contraceptive ring was seen.
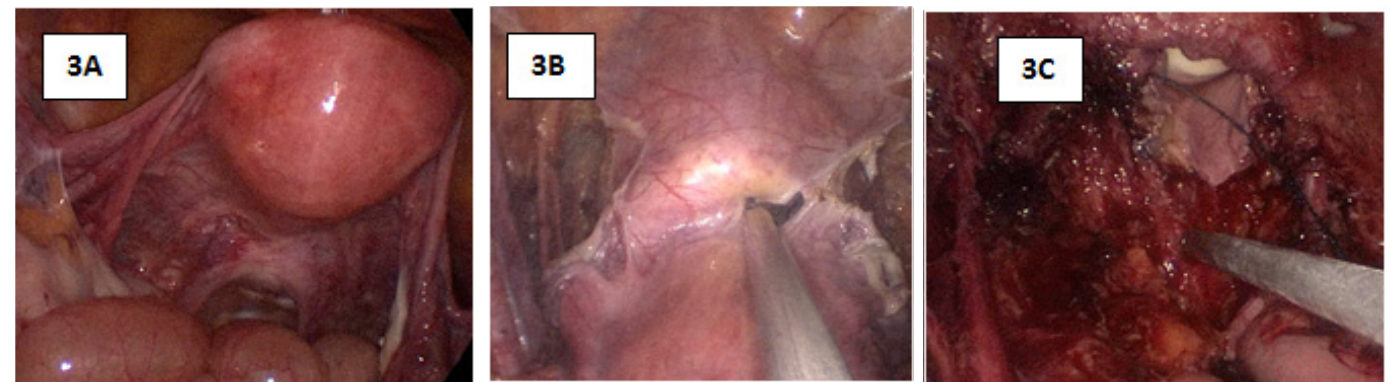

Figure 3 Intraoperative Images: 3A: there was no effusion and no adhesion in the pelvic cavity. Uterus and bilateral adnexa appeared normal.

Citation: Rezai S, Zeng C, Hughes AC, et al. Single-incision laparoscopic surgery (SILS), radical hysterectomy for early stage cervical cancer, a case report and review of literature. Obstet Gynecol Int J. 2018;9(5):37I-376. DOI: 10.15406/ogij.2018.09.00368 


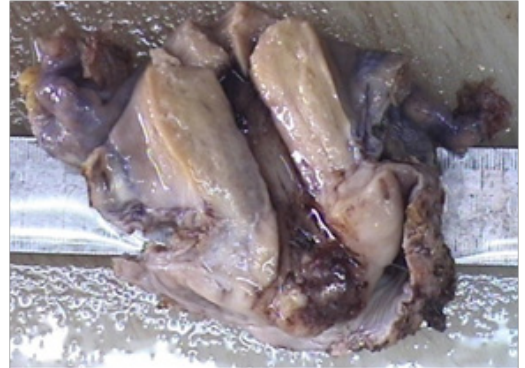

Figure 4 Cervical squamous cell carcinoma (non-keratinized), Stage IBI, Gross Pathology Specimen: normal Uterus and bilateral adnexa. Cauliflowerlike cervical mass with a size of $3 \times 2 \times 0.7 \mathrm{~cm}$ noted.
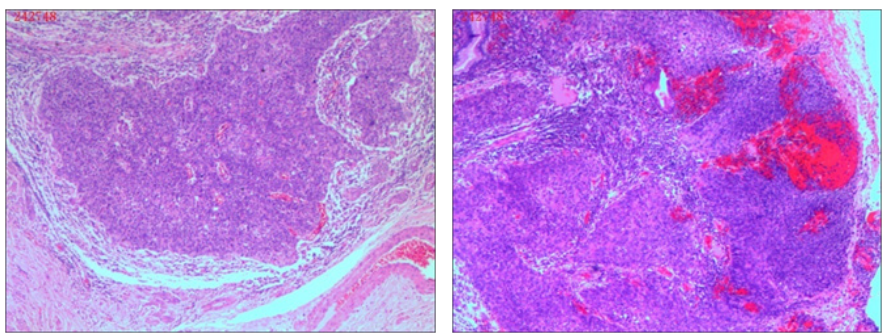

Figure 5 Pathology Slides: Examination of pathology specimen confirmed Cervical squamous cell carcinoma (non-keratinized), Stage IBI.
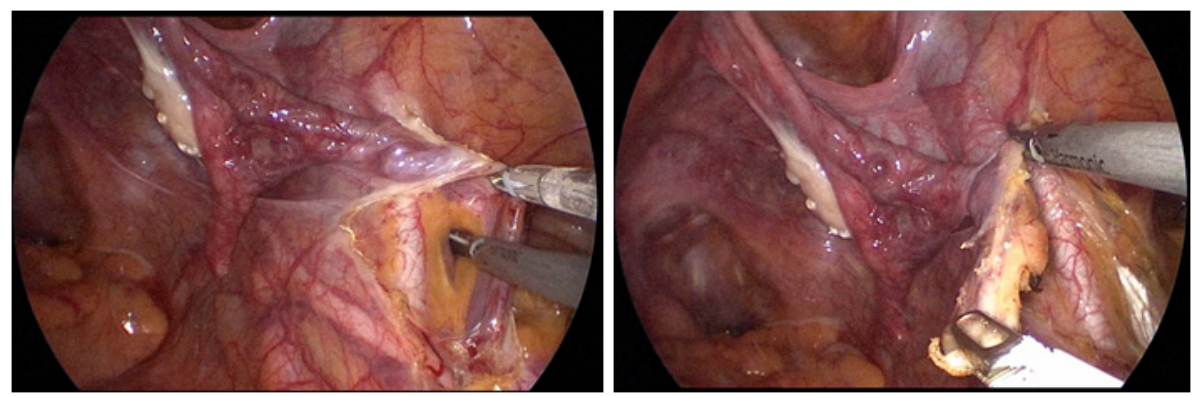

Figure 6 the right-side infundibulopelvic ligament in high position.
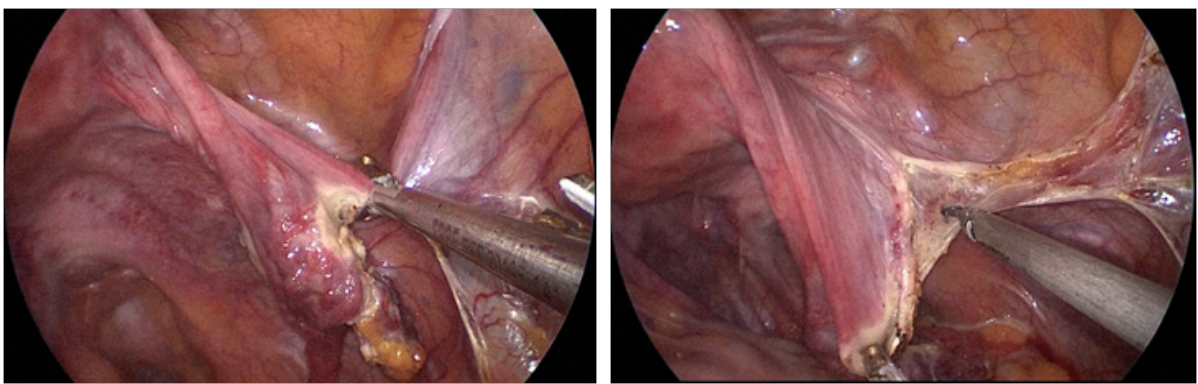

Figure 7 the right-side anterior and posterior lobes of broad ligament were opened.
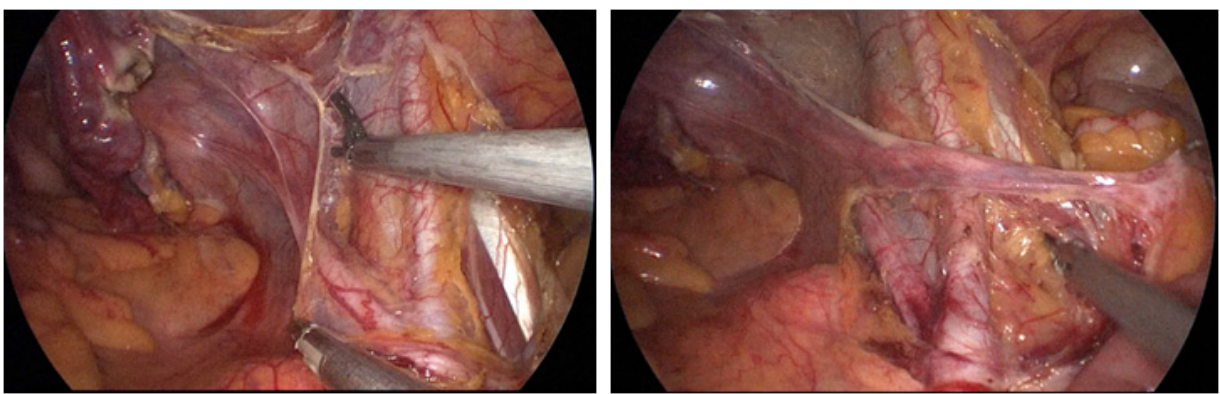

Figure $\mathbf{8}$ The lateral peritoneum was then cut to the bottom of right-hand round ligament of uterus.

Citation: Rezai S, Zeng C, Hughes AC, et al. Single-incision laparoscopic surgery (SILS), radical hysterectomy for early stage cervical cancer, a case report and review of literature. Obstet Gynecol Int J. 2018;9(5):37I-376. DOI: I0.15406/ogij.2018.09.00368 


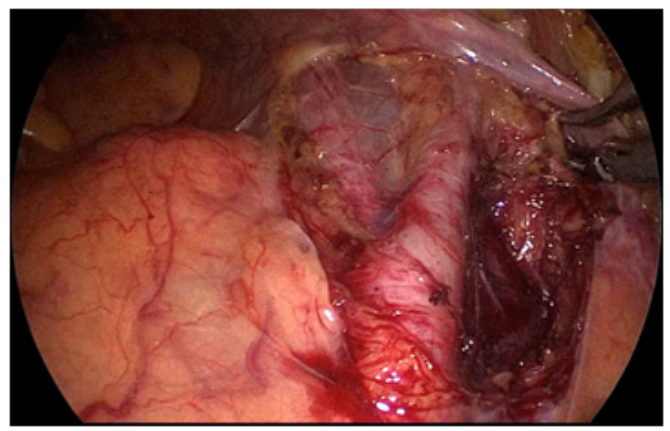

Figure 9 The lateral peritoneum was then cut to the bottom of right-hand round ligament of uterus.
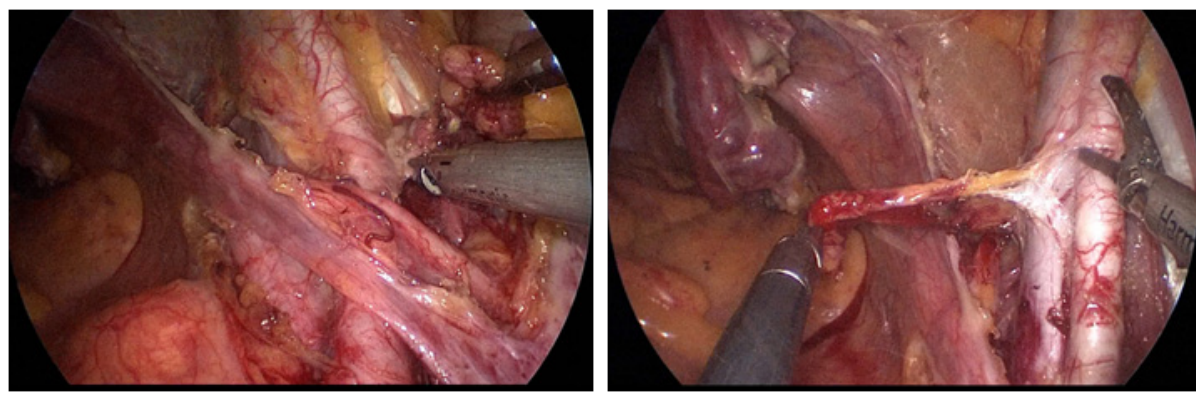

Figure 10 external iliac nodes.
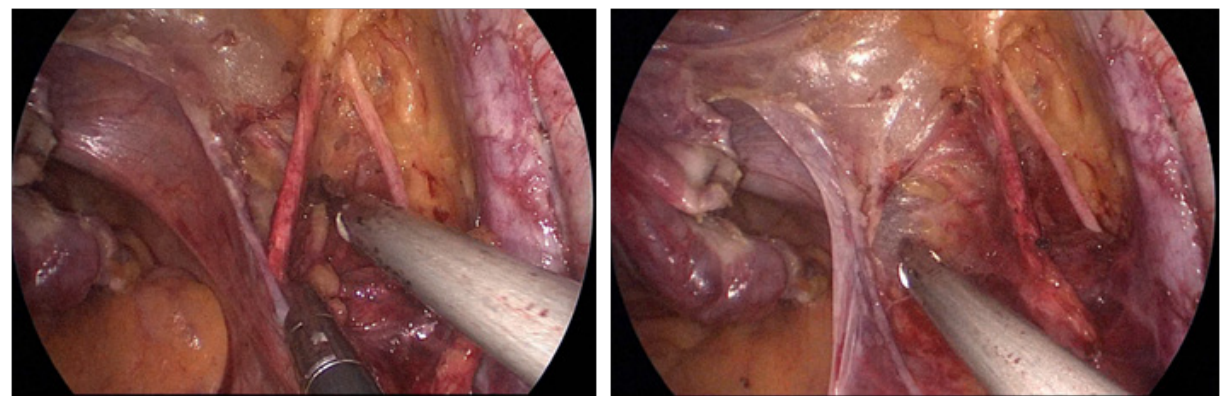

Figure I I inguinal nodes, obturator nest nodes.
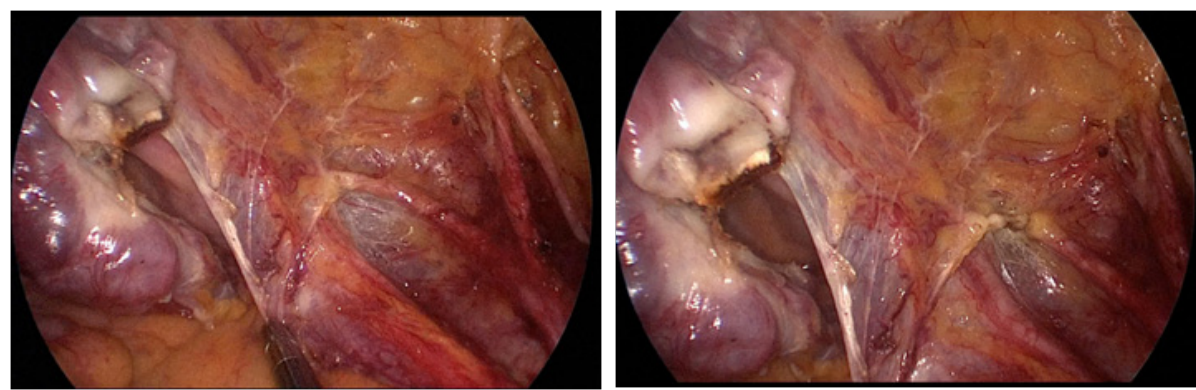

Figure 12 After lymph node dissection the right uterine artery was ligated.
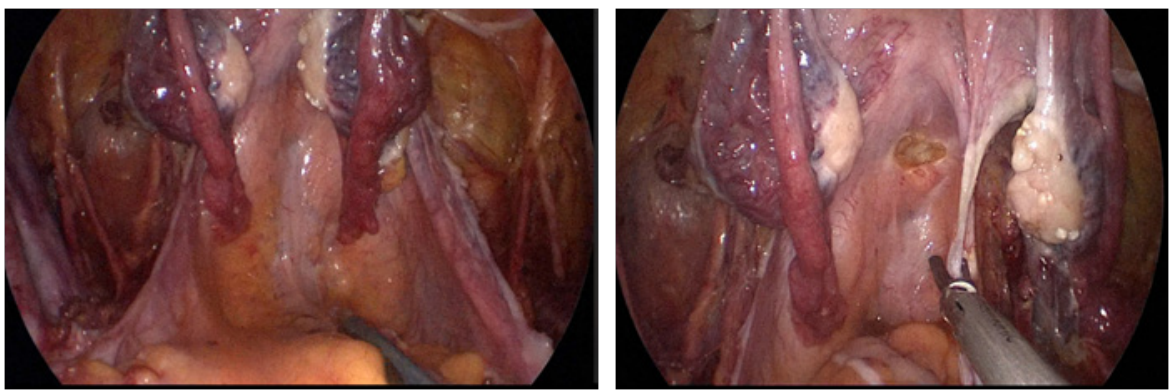

Figure 13 the rectum was separated from the posterior wall and upper segment of vagina.

Citation: Rezai S, Zeng C, Hughes AC, et al. Single-incision laparoscopic surgery (SILS), radical hysterectomy for early stage cervical cancer, a case report and review of literature. Obstet Gynecol Int J. 2018;9(5):37I-376. DOI: I0.15406/ogij.2018.09.00368 
The vesicular peritoneum reflection was exposed; we then separated loose tissue between the bladder and the cervix to reach below the external cervical orifice. This exposed the interspace of the bladder and cervix. The gap of the para vagina was separated from both sides exposing the ligaments of the bladder and cervix; the intramural segment ureter was dissociated. (Figure 14) The bilateral rectal lateral fossa and pararectal pouch was opened to expose the bi-sacral uterine and the cardinal ligaments of the uterus. The bilateral sacrouterine ligament was removed $30 \mathrm{~mm}$ from the cervix. The cardinal ligament was dissected, and the pelvic side wall, including the vagina cuff, was closed. Procedure time was four hours and thirty minutes, with an estimated blood loss of $50 \mathrm{ml}$.
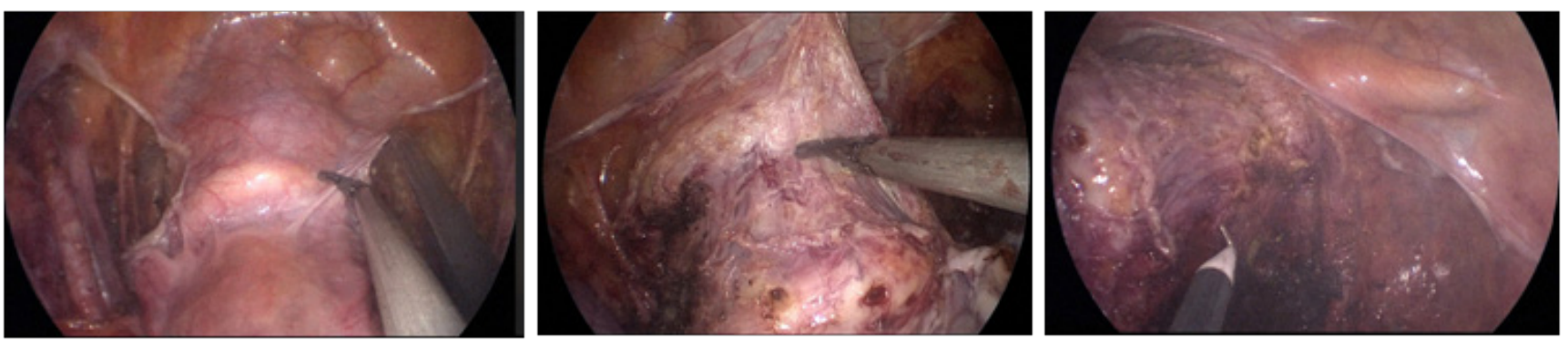

Figure I 4 The gap of the para vagina was separated from both sides exposing the ligaments of the bladder and cervix; the intramural segment ureter was dissociated.

Pathology specimen was significant for a normal uterus and bilateral adnexa with a cauliflower-like cervical mass $(3 \times 2 \times 0.7 \mathrm{~cm})$ (Figure 4). Histologic evaluation confirmed the diagnosis of cervical squamous cell carcinoma (non-keratinized), Stage IB1 (Figures 5A $\& 5 \mathrm{~B})$. The patient remained in the hospital for 10 days; three days to improve related auxiliary examinations and two days to improve bowel preparation. On the fifth day she received surgery in was discharged home in the tenth day, without complications.

\section{Discussions}

Radical hysterectomy is still the treatment of choice for most women who have early stage cervical cancer. ${ }^{11}$ Minimally invasive approaches to radical hysterectomies have been shown to be a safe alternative to traditional abdominal laparotomy. ${ }^{11}$ Minimally invasive gynecological procedures have been associated with a shorter hospital stay and improved outcomes such as less post-operative complications and improved pain control. ${ }^{4,6,12}$ SILS utilizes one incision in the umbilicus reducing the amount of abdominal incisions needed for the procedure. All instruments are introduced through the single incision utilizing a device, which allows for maintaining abdominal insufflation. ${ }^{7}, 11$ Although the challenges of crowding and instrument clashing still exist technologic advances have reduced the impact of this problem. SILS as a minimally invasive procedure specifically has the potential for a minimal scaring as the incision can be incorporated into the umbilicus. ${ }^{13}$

Another advantage of SILS compared to traditional laparoscopy specific to oncology is the reduced likelihood of seeding abdominal incisions. Although no reports have been published on seeding of trocar sites, it has been raised as a concern in the literature. With SILS only one incision is used reducing the risk of multiple seeding sites. ${ }^{6,14,15}$

Recent reports have supported the use of SILS for gynecologic procedures including cervical cancer management. But, only a few long-term studies have been conducted; however, enough evidence has not been proposed to show statistical significance when using SILS. ${ }^{11,16,17}$ In contrast, short term studies have shown equivocal outcomes for using SILS compared to standard surgical management when assessing the number of disease free years after surgery. ${ }^{4,18}$

\section{Conclusion}

Previously, cervical cancer has been managed through LEEP, cone biopsy and total abdominal hysterectomy. Typically, total abdominal hysterectomy surgery has been managed through laparotomy and laparoscopy. ${ }^{17,19}$ Based on our research, a newer more appealing procedure, SILS has been proposed as a safer alternative. ${ }^{5,20}$ SILS uses a single incision site and is associated with less scarring and shortened post-operative hospitalization. ${ }^{15,19,21}$ More studies are necessary to evaluate the short and long-term advantages for management of cervical cancer using SILS., ${ }^{4,6,17}$

\section{Acknowledgments}

Dr. Xiaoming Guan is a speaker for Applied Medical, Rancho Santa Margarita, California.

\section{Conflicts of interest}

Authors did not report any potential conflicts of interests.

\section{References}

1. Landoni F, Maneo A, Colombo A, et al. Randomized study of radical surgery versus radiotherapy for stage 1b-11a cervical cancer. Lancet. 1997;350(9077):535-540.

2. Burd EM. Human papillomavirus and cervical cancer. Clin Microbiol Rev. 2003;16(1):1-17.

3. Ratnam S, Franco EL, Ferenczy A. Human papillomavirus testing for primary screening of cervical cancer precursors. Cancer Epidemiol Biomarkers Prev. 2000;9(9):945-951.

4. Dueñas-Gonzalez A, López-Graniel C, González-Enciso A, et al. A phase II study of multimodality treatment for locally advanced cervical cancer: neoadjuvant carboplatin and paclitaxel followed by radical hysterectomy and adjuvant cisplatin chemoradiation. Ann Oncol. 2003;14(8):1278-1284.

5. Park JY, Kim YM1, Lee YY, et al Laparoendoscopic single-site radical hysterectomy for early stage cervical cancer. Obstet Gynecol Sci. 2017;60(1):110-114

6. Nam JH, Park JY, Kim DY, et al. Laparoscopic versus open radical hysterectomy in early-stage cervical cancer: long-term survival outcomes in a matched cohort study. Ann Oncol. 2012;23(4):903-911.

7. Health Quality Ontario. Robotic-assisted minimally invasive surgery for gynecologic and urologic oncology: an evidence-based analysis. Ont Health Technol Assess Ser. 2010;10(27):1-118.

8. Taylor SE, McBee WC Jr, Richard SD, et al. Radical hysterectomy for early stage cervical cancer: laparoscopy versus laparotomy. JSLS 2011;15(2):213-217. 
9. Puntambekar SP, Katakdhond S, Galgali S, et al. Duplication of Conventional Multiport Laparoscopic Radical Hysterectomy Steps with Other Minimal Access Modalities (LESS, OASIS, Robotic),. J Minim Invasive Gynecol. 2015;22(4):541-542.

10. Tergas AI, Fader AN. Laparoendoscopic single-site surgery (LESS) radical hysterectomy for the treatment of early stage cervical cancer. Gynecol Oncol. 2013;129(1):241-243.

11. Wright JD, Herzog TJ, Neugut AI, et al. Comparative effectiveness of minimally invasive and abdominal radical hysterectomy for cervical cancer. Gynecologic oncology. 2012;127(1):11-17.

12. Mori KM, Neubauer NL. Minimally invasive surgery in gynecologic oncology. ISRN obstetrics and gynecology. 2013: 312982.

13. Boruta DM, Fagotti A, Bradford LS, et al. Laparoendoscopic singlesite radical hysterectomy with pelvic lymphadenectomy: initial multiinstitutional experience for treatment of invasive cervical cancer. $J$ Minim Invasive Gynecol. 2014;21(3):394-398.

14. Fagotti A, Ghezzi F, Boruta DM, et al. Minilaparoscopic radical hysterectomy (mLPS-RH) vs laparoendoscopic single-site radical hysterectomy (LESS-RH) in early stage cervical cancer: a multicenter retrospective study. J Minim Invasive Gynecol. 2014;21(6):1005-9.
15. Puntambekar SP, Lawande A, Agarwal Joshi G, et al. Single-site laparoscopic radical hysterectomy using conventional ports and instruments. J Minim Invasive Gynecol. 2014;21(4):539.

16. Hahn HS, Kim YW. Single-port laparoscopic pelvic lymph node dissection with modified radical vaginal hysterectomy in cervical cancer. Int J Gynecol Cancer. 2010;20(8):1429-1432.

17. Barranger E, Coutant C, Cortez A, et al. Sentinel node biopsy is reliable in early-stage cervical cancer but not in locally advanced disease. Ann Oncol. 2005;16(8):1237-1242.

18. Sobiczewski P, Bidzinski M, Derlatka P, et al. Early cervical cancer managed by laparoscopy and conventional surgery: comparison of treatment results. Int J Gynecol Cancer. 2009;19(8):1390-1395.

19. Guo J, Yang L, Cai J, et al. Laparoscopic procedure compared with open radical hysterectomy with pelvic lymphadenectomy in early cervical cancer: A retrospective study. Onco Targets Ther. 2018;11:5903-5908.

20. Desai R, Puntambekar SP, Lawande A, et al. More with LESS: a novel report of nerve sparing radical hysterectomy performed using LESS. $J$ Minim Invasive Gynecol. 2013; 20(6):886-890.

21. Nam EJ, Kim SW, Lee M, et al. Robotic single-port transumbilical total hysterectomy: a pilot study. J Gynecol Oncol. 2011;22(2):120-126. 